\title{
A BOUNDARY VALUE PROBLEM ON AN INFINITE INTERVAL
}

\author{
by ATHANASSIOS G. KARTSATOS
}

(Received 22nd October 1973)

\section{Introduction}

We are interested here in proving the existence of solutions to the (generalised) boundary value problem

$$
\begin{gathered}
x^{\prime}=A(t) x+F(t, x) \\
T x=r,
\end{gathered}
$$

where $A$ is a continuous $n \times n$ matrix on $\mathbf{R}_{+}=[0, \infty), F$ is a continuous $n$ vector on $\mathbf{R}_{+} \times S$ ( $S=$ a suitable subset of $\left.\mathbf{R}^{n}\right), T$ is a bounded linear operator defined on (or on a subspace of) $C\left[\mathbf{R}_{+}, \mathbf{R}^{n}\right]$, the space of all bounded and continuous $\mathbf{R}^{n}$-valued functions on $\mathbf{R}_{+}$, and $r$ is a fixed vector in $\mathbf{R}^{n}$. There is an abundance of papers dealing with the problem ((I), (II)) on finite intervals, either in its full generality (cf., for example, (1), (2), (3), (4), (6)), or for special cases of the operator $T$. The reader is especially referred to the work of Shreve (7), (8) for such problems on infinite intervals for scalar equations. A series representation of the solutions is given by Kravchenko and Yablonskii (5). Most of our methods are extensions of the corresponding ones on finite intervals with some variations concerning the application of fixed-point theorems. Examples of interesting operators $T$ are

$$
\begin{array}{r}
T x=\int_{0}^{\infty} V(t) x(t) d t \\
T x=M x(0)+N x(\infty),
\end{array}
$$

where $V(t), M, N$ are $n \times n$ matrices with $V(t)$ integrable.

Problems of the type (I), (II) have some applications in boundary layer theory.

\section{Preliminaries}

We denote by $C$ the Banach space of all bounded and continuous $\mathbf{R}^{n}$-valued functions on $\mathbf{R}_{+}$with norm:

where $\|x\|=\sum_{i}\left|x_{l}\right|, x \in \mathbf{R}^{n}$.

$$
\|f\|_{c}=\sup _{t \in \mathbf{R}_{+}}\|f(t)\|
$$

$C^{l}$ consists of all functions $f \in C$ such that

$$
\lim _{t \rightarrow \infty} f(t) \text { exists }
$$


and is finite. $C^{l}$ is a closed subspace of $C$. For an $n \times n$ real matrix $A$ we put

$$
\|A\|=\sup _{\|x\| \leqq 1}\|A x\| .
$$

We denote by $C^{n \times n}$ the set of all continuous matrices on $R_{+}$. For a fixed positive integer $\mu$ we put $S_{\mu}=\left\{x \in \mathbf{R}^{n} ;\|x\| \leqq \mu\right\}$.

The operator $T: C \rightarrow \mathrm{R}^{n}$ (or $T: C^{\prime} \rightarrow \mathrm{R}^{n}$ ) will be assumed to be bounded and linear.

Now let $\tilde{T}$ denote the operator, which takes the matrix $A(t) \in C^{n \times n}$ into the matrix $\tilde{T} A$, whose columns are the values of $T$ at the corresponding columns of $A(t)$. Then $T$ is a bounded linear operator and $(T A) \xi=T(A(t) \xi)$ for any $A(t) \in C^{n \times n}$ and any vector $\xi \in \mathbf{R}^{n}$. Consider now the equation

$$
x^{\prime}=A(t) x+B(t)
$$

where $A \in C^{n \times n}$ and $B$ is a continuous $n$-vector defined on $\mathbf{R}_{+}$. Let $X(t)$ be the fundamental matrix of solutions of the homogeneous equation $(B \equiv 0)$ with the property $X(0)=I$, and assume that the matrix $T X$ is invertible for all $t \in \mathbf{R}$. Then the solution to the problem ((I), (II)) (which is unique) can be written as

where

$$
x(t)=X(t)[\tilde{T} X]^{-1}(r-T p)+p(t),
$$

$$
p(t)=\int_{0}^{t} X(t) X^{-1}(s) B(s) d s .
$$

For the initial condition $x(0)=x_{0}$ we obtain

$$
x_{0}=[\widetilde{T X}]^{-1}(r-T p)
$$

\section{Main results}

In the following two theorems and their corollaries $[\tilde{T X}]^{-1}$ exists. In Theorem $1, T$ is defined on $C^{l}$.

Theorem 1. For the system

assume the following:

$$
x^{\prime}=A(t) x+F(t, x)
$$

(i) $\lim _{i \rightarrow \infty} X(t)=X(\infty)$ exists and is finite;

(ii) if $_{u \in S \mu}^{\infty} \sup _{u}\left\|X^{-1}(t) F(t, u)\right\|=q(t)$, then

(iii) if

$$
N=\int_{0}^{\infty} q(s) d s<\infty
$$

$$
K_{f}=[\widetilde{T} X]^{-1}\left(r-T \int_{0}^{t} X(t) X^{-1}(s) F(s, f(s)) d s\right)
$$

for every $f \in S^{\mu}=\left\{f \in C^{l} ;\|f\|_{C} \leqq \mu\right\}$, and 
then $L M+N \leqq \mu$.

$$
\max _{f \in S^{\mu}}\left\|K_{f}\right\|=M, \max _{t \geqq 0}\|X(t)\|=L,
$$

Then there exists at least one solution to the problem ((2), (II)).

Proof. Consider the operator $U: S^{\mu} \rightarrow C^{l}$. $U$ maps the function $f \in S^{\mu}$ into the function $U f \in C^{\prime}$ given by

$$
(U f)(t)=X(t)\left[K_{f}+\int_{0}^{t} X^{-1}(s) F(s, x(s)) d s\right] .
$$

It follows from (iii) that $U S^{\mu} \subset S^{\mu}$. have

Now fix $f \in S^{\mu}$ and let $x_{f}(t) \equiv(U f)(t)$ with $\lim _{t \rightarrow \infty} x_{f}(t)=x_{f}(\infty)$. Then we

$$
\begin{aligned}
\left\|x_{f}(t)-x_{f}(\infty)\right\| & =\| X(t)\left[K_{f}+\int_{0}^{t} X^{-1}(s) F(s, f(s)) d s\right] \\
& \quad-X(\infty)\left[K_{f}+\int_{0}^{\infty} X^{-1}(s) F(s, f(s)) d s\right] \| \\
& \leqq M\|X(t)-X(\infty)\|+N\|X(t)-X(\infty)\| X(\infty) \| \int_{t}^{\infty} q(s) d s \\
& \leqq(M+N)\|X(t)-X(\infty)\|+L \int_{t}^{\infty} q(s) d s .
\end{aligned}
$$

It follows that given $\varepsilon>0$ there exists $t_{0}(\varepsilon)>0$ such that $\left\|x_{f}(t)-x_{f}(\infty)\right\|<\varepsilon$ for every $t>t_{0}(\varepsilon)$ and every $f \in S^{\mu}$. Consequently, $\left\{x_{f}\right\}, f \in S^{\mu}$ is a uniformly convergent family. Now it is easy to see that this family is equicontinuous and since it is also uniformly bounded, it is relatively compact in $C^{l}$ (cf. Kartsatos (4)). In order to show the continuity of $U$ on $S^{\mu}$, let $\left\{f_{n}\right\} \subset S^{\mu}, f \in S^{\mu}$ such that

$$
\lim _{n \rightarrow \infty}\left\|f_{n}-f\right\|_{C}=0 \text {. }
$$

Moreover, let $T f_{n}=x_{n}, T f=x$. Then we obtain

$$
\begin{aligned}
\left\|x_{n}-x\right\|_{C} & \leqq L\left[\left\|K f_{n}-K_{f}\right\|+\int_{0}^{\infty}\left\|X^{-1}(s)\left[F\left(s, f_{n}(s)\right)-F(s, f(s))\right]\right\| d s\right] \\
& \leqq L(L\|T\|+1) \int_{0}^{\infty} \| X^{-1}(s)\left[F\left(s, f_{n}(s)\right)-F(s, f(s)) \| d s\right] .
\end{aligned}
$$

The integrand in the last member of (3) tends to zero as $n \rightarrow \infty$ and is uniformly bounded by the integrable function $2 q(t)$. It follows from Lebesgue's theorem that $\lim \left\|x_{n}-x\right\|_{c}=0$. Consequently, $U$ is continuous on $S^{\mu}$ and has a fixed 
point in $S^{\mu}$ by Schauder's theorem. This fixed point is a solution to the problem ((2), (II)).

In the following theorem we make use of the representation (III).

By $x\left(t, 0, x_{0}\right)$ we denote the solution $x(t)$ of (I) with $x(0)=x_{0}$.

Theorem 2. Assume that existence, uniqueness and extendability to $[0, \infty)$ hold for the system (2) with respect to initial conditions $\left(0, x_{0}\right), x_{0} \in S_{\mu}$. Assume the existence of a constant $k>0$ such that

Let

$$
\|x(\cdot, 0, u)\|_{c} \leqq k, \quad u \in S_{\mu} .
$$

where

$$
N=\int_{0}^{\infty} q(s) d s<\infty,
$$

Then if

$$
q(t)=\sup _{\|v\| \leqq k}\left\|X^{-1}(s) F(s, v)\right\| \text {. }
$$

$\left\|[\tilde{T} X]^{-1}\right\|(r+\|T\| L N) \leqq \mu, \quad$ (where $L$ is defined as in Theorem 1), there exists at least one solution to the problem ((2), (II)).

Proof. Consider the operator $U: S_{\mu} \rightarrow S_{\mu}$ with the property:

$$
U u=[\tilde{T X}]^{-1}\left(r-T \int_{0}^{t} X(t) X^{-1}(s) F(s, x(s, 0, u)) d s\right) .
$$

We show that $U$ is continuous. In fact, let $\left\{u_{n}\right\} \subset S_{\mu}, u_{0} \in \in_{-}^{-} S$ with

$$
\begin{array}{r}
\left\|u^{n}-u^{0}\right\| \leqq\left\|[T X]^{-1}\right\|\|T\| L \int_{0}^{\infty} \| X^{-1}(s)\left[F\left(s, x\left(s, 0, u_{n}\right)\right)\right. \\
-F\left(s, x\left(s, 0, u_{0}\right)\right) \| d s .
\end{array}
$$$$
\lim _{n \rightarrow \infty}\left\|u_{n}-u_{0}\right\|=0 \text { and } u^{n}=U u_{n}, u^{0}=U u_{0}
$$

The integrand in (4) tends pointwise to zero and is bounded by the integrable function $2 q(t)$. It follows from Lebesgue's theorem that

$$
\lim _{n \rightarrow \infty}\left\|u^{n}-u^{0}\right\|=0
$$

which proves the continuity of $U$. By Brouwer's fixed-point theorem, there exists at least one vector $x_{0}$ with the property

$$
x_{0}=[\widetilde{T} X]^{-1}\left(r-T \int_{0}^{t} X(t) X^{-1}(s) F\left(s, x\left(s, 0, x_{0}\right)\right) d s\right) .
$$

The solution of (2) with initial condition $x(0)=x_{0}$ satisfies also (II).

Corollary 1. Assume that (i) of Theorem 1 holds along with following:

(i) $\lim _{n \rightarrow \infty} \inf \frac{1}{n} \int_{0}^{\infty} \sup _{\|u\| \leqq}\left\|X^{-1}(s) F(s, u)\right\| d s=0$. 
Then there exists at least one solution to the problem ((I), (II)) for every $r \in \mathbf{R}^{n}$.

Proof. Fix $r \in \mathbf{R}^{n}$ and choose a sequence $\left\{k_{n}\right\}, n=1,2, \ldots$, of natural numbers, such that

where

$$
\lim _{n \rightarrow \infty} k_{n}=\infty \text { and } \lim _{n \rightarrow \infty} \mu_{n}=0 \text {, }
$$

$$
\mu_{n}=\frac{1}{k_{n}} \int_{0}^{\infty} \sup _{\|u\| \leqq k_{n}}\left\|X^{-1}(s) F(s, u)\right\| d s .
$$

Then there exists $n_{0}$ such that

for every $n \geqslant n_{0}$.

$$
\frac{1}{k_{n}} L\left\|[\tilde{T} X]^{-1}\right\|\|r\|+L\left(L\left\|\left[T X^{-1}\right]\right\|\|T\|+1\right) \mu_{n} \leqq 1
$$

Thus,

$$
\begin{aligned}
& \| X(t)[T X]^{-1}\left(r-T \int_{0}^{t} X(t) X^{-1}(s) F(s, f(s)) d s\right) \\
&+\int_{0}^{t} X(t) X^{-1}(s) F(s, f(s)) d s \| \leqq k_{n_{0}}=N
\end{aligned}
$$

for every $f \in S^{N}=\left\{f \in C^{l} ;\|f\|_{C} \leqq N\right\}$, and the rest of the proof follows as in Theorem 1.

Corollary 2. Assume that

(i) $\|X(t)\| \leqq L, t \in \mathbf{R}_{+}$;

(ii) existence, uniqueness and extendability to $[0, \infty)$ hold for the system (2) with respect to initial conditions $\left(0, x_{0}\right), x_{0} \in \mathbf{R}^{n}$;

(iii) $\liminf _{n \rightarrow \infty} \frac{1}{n} \int_{0}^{\infty} \sup _{\|u\| \leqq n}\left\|X^{-1}(s) F(s, x(s, 0, u))\right\| d s=0$.

Then there exists at least one solution to the problem ((I), (II)) for every $r \in \mathbf{R}^{n}$.

The proof is similar to that of Corollary 1.

In the following result we give sufficient conditions for the existence of at least one value of the $\mathbf{R}^{n}$-valued parameter $\lambda$ such that the system

$$
x^{\prime}=A(t) x+B(t, x, \lambda), \quad x(0)=\zeta
$$

has a solution satisfying (II). The advantage here is that we can approximate such a solution.

Theorem 3. For the system (5) assume the following:

(i) $\left\|X^{-1}(t)\left(F\left(t, u_{1}, \lambda_{1}\right)-F\left(t, u_{2}, \lambda_{2}\right)\right)\right\| \leqq \beta(t)\left(\left\|u_{1}-u_{2}\right\|+\left\|\lambda_{1}-\lambda_{2}\right\|\right)$ for every $u_{1}, u_{2}, \lambda_{1}, \lambda_{2} \in S_{\mu}$, where $\beta(t)$ satisfies

$$
M=\int_{0}^{\infty} \beta(t) d t<\infty
$$

E.M.S. $-19 / 3-$ R 
(ii) $N=\int_{0}^{\infty} \sup _{\|\underset{\lambda}{u}\| \leqq \mu}\left\|X^{-1}(s) F(s, u, \lambda)\right\| d s<\infty$ and $L[\|\zeta\|+N] \leqq \mu$ for $a$ fixed vector $\xi \in \mathbf{R}^{n}$, where

(iii) $\left\|T X(t) \int_{0}^{t} X^{-1}(s)\left[F\left(s, x(s), \lambda_{1}\right)-F\left(s, x(s), \lambda_{2}\right)\right] d s\right\| \geqq Q\left\|\lambda_{1}-\lambda_{2}\right\|$ for

$$
L=\sup _{t \geqq 0}\|X(t)\| \text {. }
$$

every $x \in S^{\mu}=\left\{f \in C ; f(0)=\xi\right.$, $\left.\|f\|_{c} \leqq \mu\right\}$, where

$$
L M(1+\|T\| L M / Q)<1 \text {; }
$$

(iv) for each $\lambda \in S_{\mu}$ there exists a function $x_{\lambda}(t) \in S^{\mu}$ such that the solution $u_{\lambda}(t)$ of the system

$$
u^{\prime}=A(t) u+F\left(t, x_{\lambda}(t), \lambda\right)
$$

with $u_{\lambda}(0)=\xi$ satisfies $T u_{\lambda}=r$.

Then the problem ((5), (II)) has at least one solution.

Proof. Let $\lambda_{0}$ be a vector in $S_{\mu}$ and let $x_{0}(t)$ be a function in $S^{\mu}$ such that the function

$$
x_{1}(t)=X(t)\left[\zeta+\int_{0}^{t} X^{-1}(s) F\left(s, x_{0}(s), \lambda_{0}\right) d s\right]
$$

satisfies $T x_{1}=r$. It follows from (ii) that $x_{1}(t)$ belongs to $S^{\mu}$. Similarly, define by induction

$$
x_{n}(t)=X(t)\left[\zeta+\int_{0}^{t} X^{-1}(s) F\left(s, x_{n-1}(s), \lambda_{n-1}\right) d s\right]
$$

where $\lambda_{n-1} \in S^{\mu}, x_{n-1} \in S^{\mu}$ and $T x_{n}=r$. Then we have

\section{Moreover}

$$
\left\|x_{n}-x_{n-1}\right\|_{c} \leqq L M\left(\left\|x_{n-1}-x_{n-2}\right\|_{C}+\left\|\lambda_{n-1}-\lambda_{n-2}\right\|\right) \text {. }
$$

$$
\begin{aligned}
0=\| T X(t) \int_{0}^{t} X^{-1}(s) & {\left[F\left(s, x_{n-1}(s), \lambda_{n-1}\right)-F\left(s, x_{n-1}(s), \lambda_{n-2}\right)\right] d s } \\
+ & T X(t) \int_{0}^{t} X^{-1}(s)\left[F\left(s, x_{n-1}, \lambda_{n-2}\right)-F\left(s, x_{n-2}, \lambda_{n-2}\right)\right] d s \|
\end{aligned}
$$

$\geqq Q\left\|\lambda_{n-1}-\lambda_{n-2}\right\|-\|T\| L M\left\|x_{n-1}-x_{n-2}\right\|_{c}$ or

$$
\left\|\lambda_{n-1}-\lambda_{n-2}\right\| \leqq(\|T\| L M / Q)\left\|x_{n-1}-x_{n-2}\right\|_{c} .
$$

Combining (6) and (7) we obtain

$$
\begin{aligned}
\left\|x_{n}-x_{n-1}\right\|_{C} & \leqq L M(1+\|T\| L M / Q)\left\|x_{n-1}-x_{n-2}\right\|_{C} \\
& \leqq(L M)^{2}(1+\|T\| L M / Q)^{2}\left\|x_{n-2}-x_{n-3}\right\|_{C} \\
& \leqq \ldots \\
& \leqq(L M)^{n-1}(1+\|T\| L M / Q)^{n-1}\left\|x_{1}-x_{0}\right\|_{C} .
\end{aligned}
$$


Consequently,

and, from (7),

$$
\lim _{n \rightarrow \infty}\left\|x_{n}-x\right\|_{c}=0
$$

$$
\lim _{n \rightarrow \infty}\left\|\lambda_{n-1}-\lambda\right\|=0,
$$

where $x \in S^{\mu}, x(0)=\xi$ and $\lambda \in S_{\mu}$.

Now let

$$
u(t)=X(t)\left[\zeta+\int_{0}^{t} X^{-1}(s) F(s, x(s), \lambda) d s\right] .
$$

Then an application of Lebesgue's theorem shows that

$$
\lim _{n \rightarrow \infty}\left\|x_{n}-u\right\|_{C}=0,
$$

which shows that $u(t) \equiv x(t), t \in \mathbf{R}_{+}$, and completes the proof of the theorem.

\section{Discussion-Example}

Suitable Lipschitz conditions on the vector $F(t, x)$ can easily guarantee the uniqueness of the solutions, whose existence is shown by Theorems 1,2 and their corollaries. It would be interesting to know the analogues of Theorems 1 and 2 in the case of non-invertibility of the matrix $\tilde{T} X$. It is also important to extend the results of this paper to systems of the form

$$
x^{\prime}=A(t, x) x+F(t, x)
$$

which have been studied, with respect to boundary value problems on finite intervals, by Opial (6), Avramescu (1), Kartsatos (4).

The following problem satisfies all the assumptions of Corollary 1 :

Here we have

$$
\begin{gathered}
x_{1}^{\prime}=-x_{1}-e^{t} x_{2}+e^{-2 t} x_{1}^{1 / 3} \\
x_{2}^{\prime}=-x_{2}+e^{-t^{2}\left(x_{1}^{3 / 5}+x_{2}^{2 / 3}\right)} \\
T x=\left[\begin{array}{ll}
1 & 2 \\
0 & 1
\end{array}\right]\left[\begin{array}{l}
x_{1}(0) \\
x_{2}(0)
\end{array}\right]+\left[\begin{array}{rr}
1 & 1 \\
-1 & 0
\end{array}\right]\left[\begin{array}{l}
x_{1}(\infty) \\
x_{1}(\infty)
\end{array}\right]=\left[\begin{array}{l}
1 \\
2
\end{array}\right] .
\end{gathered}
$$

$$
\begin{gathered}
A(t)=\left[\begin{array}{rr}
-1 & -e^{t} \\
0 & -1
\end{array}\right], \quad \tilde{T} X=\left[\begin{array}{ll}
1 & 1 \\
0 & 1
\end{array}\right] \\
X^{-1}(t)=\left[\begin{array}{cc}
e^{-t} & e^{-t}-1 \\
0 & e^{-t}
\end{array}\right]
\end{gathered}
$$

\section{REFERENCES}

(1) C. Avramescu, Sur l'éxistence des solutions pour un problème aux limites général, Ann. Mat. Pura ed Appl. 82 (1962), 69-81.

(2) C. Corduneanu, Problèmes aux limites lineaires, Ann. Mat. Pura ed. Appl. 68 (1966), 65-73. 
(3) L. N. ESukov, On a functional problem for ordinary differential equations, Uspehi Mat. Nauk 13 (1958), 191-196.

(4) A. G. KartSatos, Nonzero solutions to boundary value problems for nonlinear systems, Pacific J. Math. (to a ppear).

(5) T. K. Kravchenko and A. I. Yablonskif, Solution of an infinite boundary value problem for a third order equation, Differentsial'nye Uravnenija 1 (1965), 327 329.

(6) Z. OpIAL, Linear problems for systems of nonlinear differential equations, J. Diff. Equations 3 (1967), 580-594.

(7) W. E. Shreve, Boundary value problem for $y^{n}=\mathrm{f}(\mathrm{x}, \mathrm{y}, \lambda)$ on $[a, \infty)$, SIAM J. Appl. Math. 17 (1969), 84-97.

(8) W. E. SHREVE, Terminal value problems for second order nonlinear differential equations, SIAM J. Appl. Math. 18 (1970), 783-791.

\section{UNIVERSITY OF SOUTH FLORIDA}

TAMPA

FLORIDA 33620 